\begin{tabular}{|c|c|}
\hline Title & A Computer A ided Education System Based on A ugmented Reality by Immersion to 3-D Magnetic Field \\
\hline Author(s) & Matsutomo, Shinya; Manabe, Tomohisa; Cingoski, V latko; Noguchi, So \\
\hline Citation & $\begin{array}{l}\text { IEEE transactions on magnetics, 53(6), } 8102004 \\
\text { https://doi.org/10.1109/T MA G.2017.2665563 }\end{array}$ \\
\hline Issue Date & 2017-06 \\
\hline Doc URL & http://hdl.handle.net/2115/66969 \\
\hline Rights & $\begin{array}{l}\text { (c) } 2017 \text { IEEE. Personal use of this material is permitted. Permission from IEEE must be obtained for all other uses, in } \\
\text { any current or future media, including reprinting/republishing this material for advertising or promotional purposes, } \\
\text { creating new collective works, for resale or redistribution to servers or lists, or reuse of any copyrighted component of } \\
\text { this work in other works. }\end{array}$ \\
\hline Type & article (author version) \\
\hline File Information & 3DAR_fullpaper_final.pdf \\
\hline
\end{tabular}

Instructions for use 


\title{
A computer aided education system based on augmented reality by immersion to 3-D magnetic field
}

Shinya Matsutomo ${ }^{1}$, Tomohisa Manabe ${ }^{1}$, Vlatko Cingoski ${ }^{2}$, and So Noguchi ${ }^{3}$

${ }^{1}$ National Institute of Technology, Niihama College, Niihama 792-8580, Japan

${ }^{2}$ Faculty of Electrical Engineering, University “Goce Delcev” - Stip, Skopje, 1000, Macedonia

${ }^{3}$ Graduate School of Information Science and Technology, Hokkaido University, Sapporo 060-0814, Japan

\begin{abstract}
An immersive real-time visualization system of $3 \mathrm{D}$ magnetic field for educational purposes is presented. This immersive visualization system is based on augmented reality technology. The proposed system provides observation of a magnetic field distribution and its stereoscopic vision in 3D space using head mounted display. To improve the visualization capabilities, a new realtime method for drawing magnetic flux lines in 3D space is developed and presented in this paper. It enables a user to easily observe and grasp a magnetic field generated by multiple sources (e.g., magnets and/or multiple coils) in an augmented 3D space. Additionally, it permits a user to freely and interactively move the magnetic sources within the visualization space and to observe the magnetic fields interference in real-time. As a result, one can intuitively and easy visualize, observe and grasp the magnetic field even in $3 \mathrm{D}$ space.
\end{abstract}

Index Terms - Augmented reality technology, Electromagnetic field analysis, Visualization.

\section{INTRODUCTION}

$I_{(}^{\mathrm{r}}$ N RECENT YEARS, information and communication devices $\mathbf{I}_{\text {(e.g., smartphone, tablet computer, wearable device, head }}$ mounted display, etc.) are becoming extremely popular, and many lectures have begun using these devices widely as an education tool [1]. The major obstacle for young students' studying electromagnetic fields, is being unsuccessful to understand or imagine electromagnetic phenomena because these fields are invisible and complicated in their physical nature. Therefore, the visualization of electromagnetic field is extremely important and valuable for the students to learn and grasp a "magnetic field distribution" generated by any electromagnetic sources (e.g., magnets and/or coils). This paper deals with the development of a new tool specially designed for visualization of complex electromagnetic field phenomena in 3D space based on real-time visualization and mostly for educational purposes.

Some kinds of visualization systems for magnetic field using augmented reality technology have been reported by a several researchers [2-3]. However, they have not discussed the real-time processing of the visualization system with the magnetic field interference in details. In our previous study, a real-time visualization system which utilized an augmented reality technology for magnetic field visualization in 2D space has already been reported [4-5]. It was resented a synthetic image of mocks and their simulated magnetic field to users in 2D monitor, even interfering the magnetic field distributions of several sources. However, this method was restricted to visualize the magnetic field distributions only in 2D space.

In this paper, we propose an immersive 3D visualization system that combines a real experiment and a computer simulation utilizing the augmented reality technique [6-7] and a head mounted display (HMD). The conceptual idea behind the developed system is shown in Fig. 1. The proposed system indicates a synthetic image of objects (source materials) captured by a video camera and their simulated magnetic field to a user. In the system, a real object is not mandatory, however a simple marker which is registered to the system as preprocess is compulsory. The developed stereo 3D visualization system can assist the user to easily understand the magnetic field distribution in an "immersive augmented real world in 3D space with stereo vision." In addition, the proposed visualization system has a user-friendly interface enabling one to freely and interactively move mock objects in the $3 \mathrm{D}$ visualization space.

\section{The PROPOSED IMMERSIVE VisuALIZATION SYSTEM}

The main technical components of the proposed visualization system are: (i) the image-recognition, (ii) the computation of magnetic field distribution, and (iii) the imageprocessing for stereoscopic vision, while the main procedure of the system is as follows:
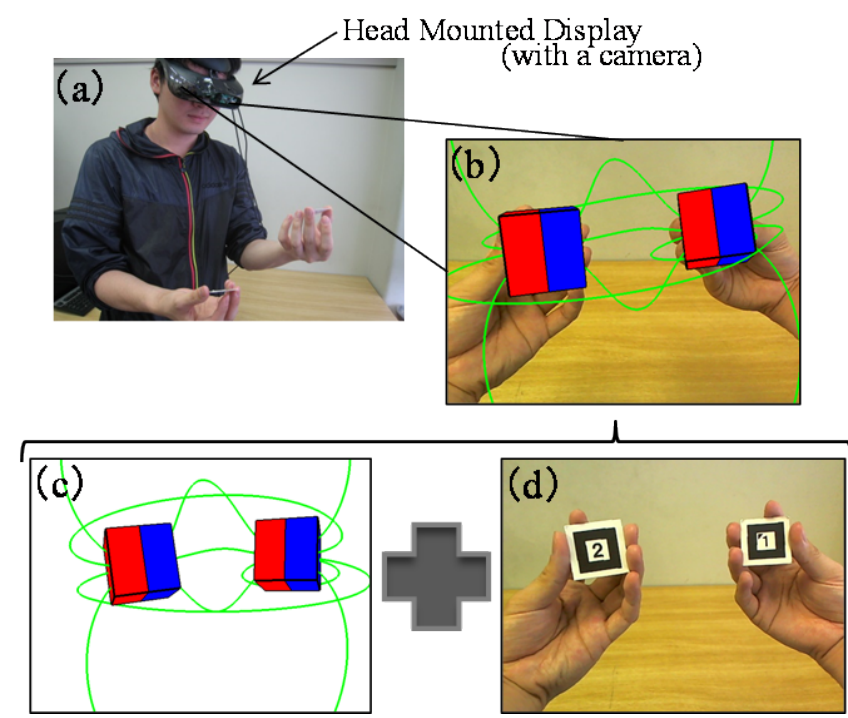

Fig. 1. The conceptual idea of developed immersive real-time visualization system for 3D magnetic field distribution. (a) Overview of proposed system and the HMD with a camera. (b) The composite image displayed on the user's HMD. (c) The simulated magnetic flux lines in 3D space. (d) The markers which indicate the positions and the type of the objects (e.g., magnet, coil, iron). 
Step 0: The marker for an object (e.g., magnet, coil, iron) is registered to the visualization system as preprocess.

Step 1: The registered markers are captured by a digital web camera attached to HMD.

Step 2: The captured markers are identified using the imagerecognition technique.

Step 3: The magnetic field distribution, which is generated by the identified markers, is computed.

Step 4: The parallax image is generated for stereoscopic vision with HMD.

Step 5: The synthetic image of the computed magnetic field distribution and the captured picture by a digital web camera are visualized simultaneously to user's HMD.

\section{A. Recognition of a Marker (Steps 0, 1, and 2)}

The marker indicates a position and a type of the object (e.g., magnet or iron). The image recognition of the makers is executed by using ARToolKit [7] in real-time. ARToolKit is an open-software library for augmented reality applications. The distance from the camera to the marker and the maker's angle can also be detected by using ARToolKit in real-time.

\section{B. Computation of magnetic field (Steps 3 and 4)}

It is important to notice that in the proposed visualization system the magnetic flux lines are always drawn in real-time, even if a user interactively moves a marker freely. Therefore, it is almost impossible to perform a real-time calculations using finite element method, because it takes a large calculation time. Therefore, the Biot-Savart's calculation method [8] and the magnetic moment method [9] were adopted for computation in the proposed visualization method.

The magnetic flux density $\boldsymbol{B}_{\text {coil }}$ generated by a coil with a current density $\boldsymbol{J}$ and $\boldsymbol{B}_{\text {magnet }}$ generated by a permanent magnet with magnetization $\boldsymbol{M}_{\text {magnet }}$ at a given field point according the Biot-Savart's law are given as follows:

$$
\begin{aligned}
& \boldsymbol{B}_{\text {coil }}=\frac{\mu_{0}}{4 \pi} \boldsymbol{J} \times \int_{V} \nabla\left(\frac{1}{r}\right) d V \\
& \boldsymbol{B}_{\text {magnet }}=-\frac{1}{4 \pi} \nabla\left\{\int_{V} \boldsymbol{M}_{\text {magnet }} \cdot \nabla\left(\frac{1}{r}\right) d V\right\}+\boldsymbol{M}_{\text {magnet }}
\end{aligned}
$$

where $r$ is distance between the source point and the field point.

Furthermore, when a magnetic body exists in the space, its magnetic body could be divided into cubes and the magnetizations $\boldsymbol{M}_{\text {iron }}$ of each cube could be calculated by means of the magnetic moment method. Thus, the magnetic flux density $\boldsymbol{B}_{\text {iron }}$ similarly could be calculated as follows:

$$
\boldsymbol{B}_{\text {iron }}=-\frac{1}{4 \pi} \nabla\left\{\int_{V} \boldsymbol{M}_{\text {iron }} \cdot \nabla\left(\frac{1}{r}\right) d V\right\}+\boldsymbol{M}_{\text {iron }}
$$

To obtain the magnetizations $\boldsymbol{M}_{\text {iron }}$, one should simultaneously solve a system of linear equations generated by each cube, which could also be very time consuming calculation process. Therefore in our system, to provide a realtime visualization, the numbers of unknowns is limited.

Finally, the magnetic flux density $\boldsymbol{B}_{t}$ at a field point is calculated by summing the computational result obtained by means of (1), (2) and (3).

Next, a method to calculate a magnetic flux line is described. In 3D space, a magnetic flux line equation [10] is given as follows:

$$
\frac{d x}{B_{x}}=\frac{d y}{B_{y}}=\frac{d z}{B_{z}}
$$

where $B_{x}, B_{y}$, and $B_{z}$ are the $x$-, $y$-, and $z$-component of the magnetic flux density vector, respectively. As previously said, in order to decrease the computation time, the magnetic flux density vector is the proposed system is obtained by means of the Biot-Savart law and the magnetic moment method.

Furthermore, the virtual particle tracking method is adopted for drawing a magnetic flux line in 3D space. A single virtual particle initially is placed e.g. on the $\mathrm{N}$ pole of a magnet, and then such particle tracks the calculated magnetic density vector in the 3D space. A virtual particle position at step $t+1$, $\boldsymbol{P}_{t+1}$ is calculated from the following equation:

$\boldsymbol{P}_{t+1}=\boldsymbol{P}_{t}+\left(\frac{\boldsymbol{B}_{t}}{\left|\boldsymbol{B}_{t}\right|}\right) \Delta l$

where $\boldsymbol{P}_{t}$ is the position of that particle at step $t$, while $\Delta l$ is the movement distance of that particle at this step. If $\Delta l$ is shorter, the computation accuracy enhances although the computation time increases. Opposite, if $\Delta l$ is longer, although the computation time decreases, the virtual particle might shift out of the correct flux line, as shown in Fig. 2.

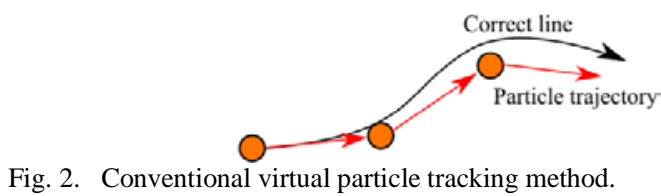

To take into account trade-off between the longer computation time on one side and the accuracy of computation, on the other, the developed system implements an adjusting method for the length of $\Delta l$. The length of $\Delta l$ is automatically shorter when the direction of the flux density changes abruptly, and $\Delta l$ becomes longer when the magnetic flux direction changes smoothly, as shown in Fig. 3.

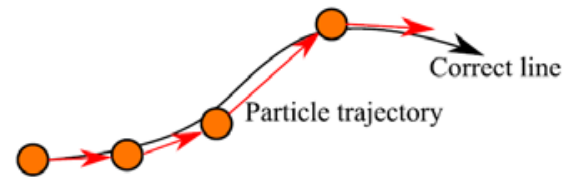

Fig. 3. Proposed virtual particle tracking method. A virtual particle correctly traces on flux line as a result of adjustable $\Delta l$ length. 
To achieve adequate adjustment of $\Delta l$ length, the following equation is applied:

$$
\boldsymbol{P}_{t+1}=\boldsymbol{P}_{t}+\left(\frac{\boldsymbol{B}_{t}}{\left|\boldsymbol{B}_{t}\right|}\right) k_{t} \Delta l
$$

where $k_{t}$ is the distance adjustment parameter as follows:

$$
k_{t}=\left\{\begin{array}{lr}
\alpha & r_{t} \geq \beta \\
\alpha+(1-\alpha)\left(1-\frac{r_{t}}{\beta}\right) & \text { otherwise }
\end{array}\right.
$$

where $r_{t}$ estimates the direction change ratio of the vector $\boldsymbol{B}$ according Fig. 4:

$$
r_{t}=\frac{1}{k_{t-1} \Delta l} \cos ^{-1}\left(\frac{\boldsymbol{B}_{t}}{\left|\boldsymbol{B}_{t}\right|} \cdot \frac{\boldsymbol{B}_{t-1}}{\left|\boldsymbol{B}_{t-1}\right|}\right)
$$

where $\alpha$ is the minimum ratio of distance $\Delta l \quad(0<\alpha \leq 1)$, and $\beta$ is the threshold value of the change ratio.

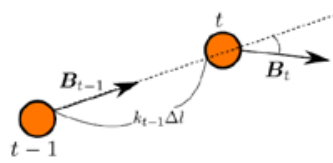

Fig. 4. Calculation of the direction change ratio for a virtual particle.

Since the proposed visualization method uses the BiotSavart's law, it is not necessary to calculate the distribution of the magnetic flux density vector for the whole domain, rather only at the observation point. As a result, this combine procedure based on the computation of magnetic flux density vector using the Biot-Savart's method and the magnetic moment method, and the virtual particle method for flux line visualization enables realization of a true real-time visualization system in 3D space.

\section{Image-processing for Stereoscopic Vision (Steps 4 and 5)}

As described above, although the magnetic flux lines could be calculated in the $3 \mathrm{D}$ space, it is difficult to enable easy observation of those magnetic flux lines in the 3D space because one cannot judge the real 3D physical behaviour of the magnetic flux line by simply using an ordinary 2D display. As a solution, our system adopted the binocular stereoscopic visualization technique as shown in Fig. 5. The system consists of a web camera and head mount display (HMD) fully compatible for 3D image processing.

Initially, as a preprocessing the original image is captured by a web camera which is attached on the HMD. Next, the magnetic flux lines are calculated and two separate images, for right and for left eye, respectively are produced. Those images are unified into one image by a side-by-side method and sent to HMD. The side-by-side method combines two images with compressed resolution to one, half of each side (left and right), and transmit to HMD as one frame.

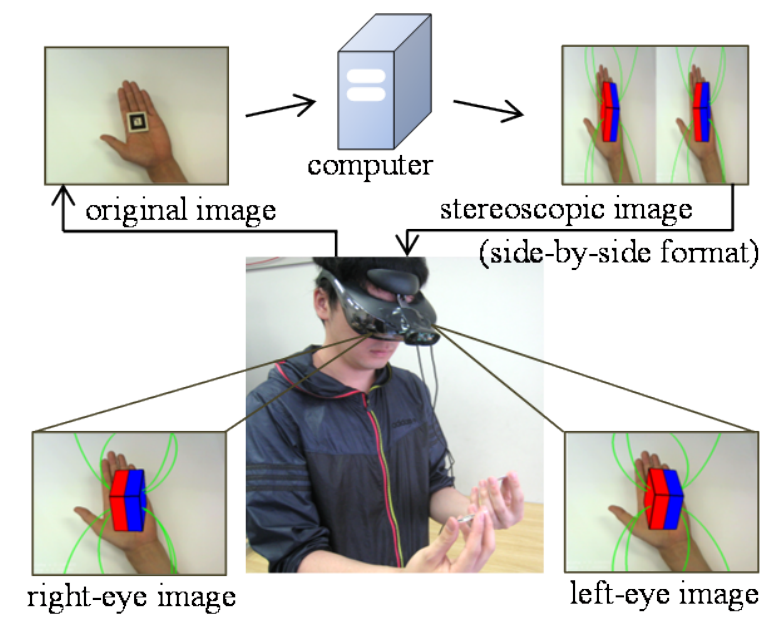

Fig. 5. The component of the binocular stereoscopic 3D visualization system. Initially, the original image is captured by web camera attached on the HMD. Next, the magnetic flux lines are calculated and two separate images, for right eye and for left eye are produced. These images are unified by a side-by-side method and are sent to HMD as a single stereoscopic image.

\section{ApplicAtion of The Proposed System}

In this section an example of the proposed system is presented. Fig. 6 shows the working environment of the developed visualization system. In Fig. 6 (a), $k_{t}$ is fixed to 0.5, and in Fig. 6 (b), to 0.1, respectively i.e., $\Delta l$ is set equal to the length of the magnet. In Fig. 6 (a), although the average computation time was very short enabling a full 23.0 fps (frames per second), the magnetic flux lines appear not natural and wrong. In Fig. 6 (b), the average computation time was little longer enabling 10.3 fps. However, in this case the magnetic flux lines appear natural and correct.

Fully implementing the proposed method with adjustable $\Delta l$ lengths, one could obtain the results presented in Fig. 6 (c) ( $\alpha=0.1, \beta=\pi / 90$ ), with the average calculation time of 23.0 fps. In Fig. 6 (c), the color of the flux lines indicates the length of the product $k_{t} \Delta l$ (i.e., red means short and blue means long). Consequently, by using our proposed method, it is possible to establish a good balance between the computation time and the accuracy of the visual simulation. Finally, our developed visualization system has some additional features, such as visualization of magnetic field interference between different magnetic sources or objects, e.g. between permanent magnet and an iron core (Fig. 7), utilization of the cone plot visualization (Fig. 8), and real-time visualization of the flux lines even in case of free and interactive movement of any object within the visualization 3D space. 


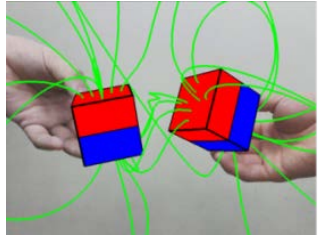

(a) $k_{t}=0.5$.

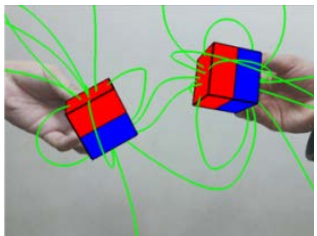

(b) $k_{t}=0.1$.

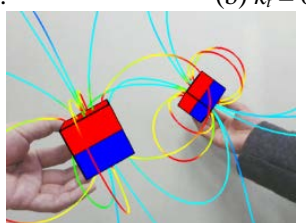

(c) Proposed method.

Fig. 6. Obtained results by the newly developed visualization system for a model with two permanent magnets.

\section{CONCLUSION}

In this paper an immersive real-time visualization system of 3D magnetic fields utilizing augmented reality technology suitable for educational purposes is presented. By utilizing the proposed visualization method, one can easily observe and perceive in real-time the magnetic field distribution generated by multiple sources (e.g. permanent magnets and/or coils), or by single source and other magnetic or nonmagnetic object in an augmented 3D space. Additionally, the user can move sources or objects interactively and observe the magnetic field distribution changes in real-time, providing intuitively observation, analysis and understandings of the physical phenomena of the magnetic field in 3D space.

\section{REFERENCES}

[1] M.E.C. Santos, A. Chen, T. Taketomi, G. Yamamoto J. Miyazaki, and H. Kato, "Augmented Reality Learning Experiences: Survey of Prototype Design and Evaluation," IEEE Transactions on Learning Technologies, Volume: 7, no.1, pp. 38 - 56, 2014.

[2] André Buchau, Wolfgang M. Rucker, Uwe Wössner, and Martin Becker, "Augmented reality in teaching of electrodynamics," COMPEL - The international journal for computation and mathematics in electrical and electronic engineering, vol. 28 no. 4, pp. 948-963, 2009.

[3] D. Buendgens, A. Hamacher, M. Hafner, T. Kuhlen, and K. Hameyer, "Bidirectional Coupling Between 3-D Field Simulation and Immersive Visualization Systems," IEEE Trans. Magn., vol. 48, no. 2, pp. 547-550, Feb. 2012.

[4] Shinya Matsutomo, Takenori Miyauchi, So Noguchi, and Hideo Yamashita, "Real-Time Visualization System of Magnetic Field Utilizing Augmented Reality Technology for Education,” IEEE Trans. Magn., vol. 48, no. 2, pp. 531-534, 2012.

[5] Shinya Matsutomo, Kenta Mitsufuji, Yuta Hiasa, and So Noguchi, "Real Time Simulation Method of Magnetic Field for Visualization System With Augmented Reality Technology,” IEEE Trans. Magn., vol. 49, no. 5, pp. 1665-1668, May 2013.

[6] R.T. Azuma, "A Survey of Augmented Reality," PresenceTeleoperators and Virtual Environments, vol. 6, no. 4, pp. 355-385, 1997

[7] H. Kato and M. Billinghurst, "Marker tracking and HMD calibration for a video-based augmented reality conferencing system," Proc. the 2nd IEEE and ACM Int. Workshop on Augmented Reality (IWAR 99), 1999.

[8] J. A. Stratton, “Electromagnetic Theory,” McGraw-Hill, 1941.

[9] F. Janet, J.-L. Coulomb, C. ChilletSo, and P. Mas, "Simplified magnetic moment method applied to current transformer modeling," IEEE Trans. Magn., vol. 40, no. 2, pp. 818-821, 2004.

[10] Takahiro Yoshigai, So Noguchi, and Hideo Yamashita, "Analytical Computation and Visualization of Magnetic Flux Lines in 3-D Space from Hexahedral Edge Finite Element Result,” IEEE Trans. Magn., vol. 41, no. 5, pp. 1820-1823, 2005.
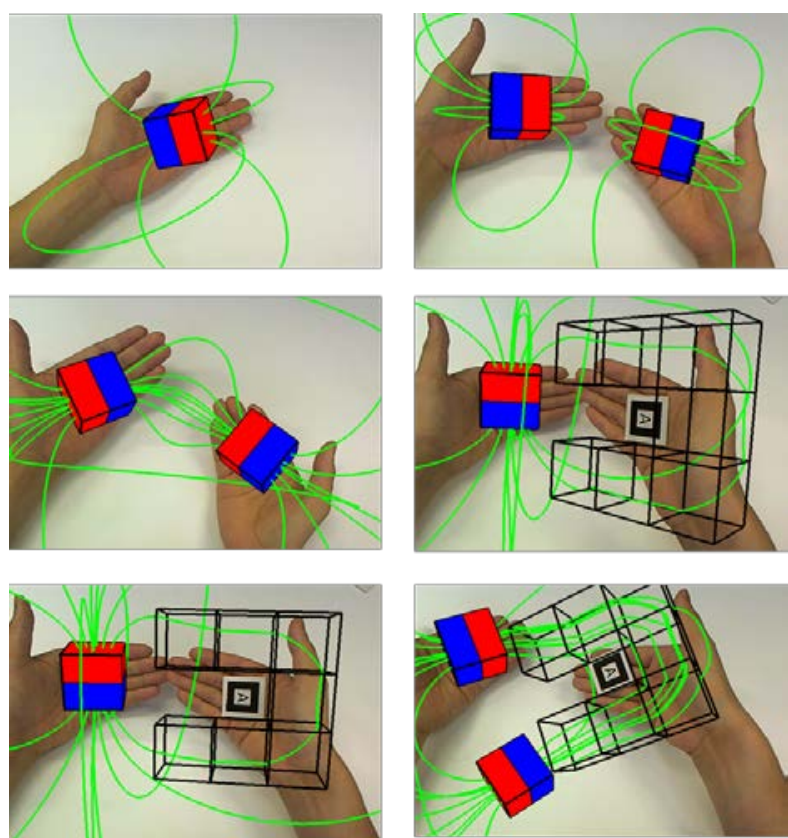

Fig. 7. Obtained results - models with two permanent magnets and permanent magnet and C-shaped iron core.
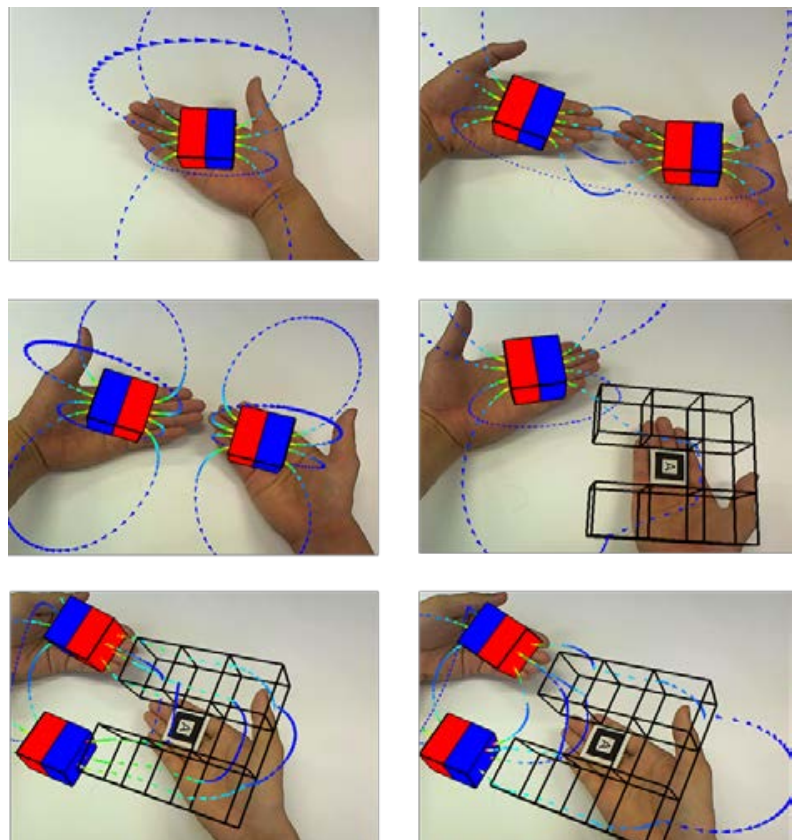

Fig. 8. Visualization of the obtained results using the cone plot visualization method. 